\title{
Quasar jets and their fields
}

\author{
BY ROGER D. BLANDFORD \\ Department of Theoretical Astrophysics, California Institute of Technology, \\ 1200 E. California Boulevard, Pasadena, CA 91125, USA
}

Observations of jets from quasars and other types of accreting black holes are briefly summarized. The importance of beaming and gamma-ray observations for understanding the origin of these jets is emphasized. It is argued that both the power source and the collimation are likely to be magnetic in origin, although the details remain controversial. Ultrarelativistic jets may be formed by the spinning hole and collimated by a hydromagnetic disc wind. Progress in understanding jets has been handicapped by our inadequate knowledge of how the magnetic field really behaves under cosmic conditions. Fortunately, significant insights are coming from solar observations, numerical simulations and laboratory plasma experiments. Some possible evolutionary ramifications are briefly discussed and it is suggested that it is the mass of the black hole relative to that of the galaxy which determines the eventual galaxy morphology.

Keywords: quasars; accretion discs; magnetic fields; jets

\section{Quasars, Seyferts and extragalactic radio sources}

\section{(a) Quasars}

When the Universe was about one-quarter of its present age, the nucleus of roughly one out of every 500 bright galaxies (at any time) was so bright that it outshone the surrounding stars. For historical reasons these nuclei are called quasi-stellar objects, or quasars for short. These quasars are generally recognized by their unusually blue optical continua and resulting broad emission lines. They also are powerful X-ray sources. Roughly $10 \%$ of these quasars are designated 'radio-loud', like the first example discovered, 3C 273, because they also possess powerful radio sources; the remainder are 'radio-quiet' (though not silent). The separation between these two classes is pretty clean, with relatively few intermediate cases. Roughly $10 \%$ of the radio-quiet quasars also exhibit broad absorption lines and are called BALQs. (For more detailed discussion of much of what follows as well as an extensive bibliography of original references that cannot be reproduced here, see, for example, Krolik (1998) and Robson (1996).)

Radio-loud quasars are further subdivided into 'compact' and 'extended' radio sources. The former group are dominated by flat spectrum radio nuclei that dominate the emission at centimetre wavelengths. The extended sources invariably comprise two 'lobes' of steep spectrum radio emission straddling the Galaxy and located beyond the observed stars. We now know that, when observed with greater sensitivity and particularly at lower frequency, the compact sources also have extended components, and, correspondingly, the extended radio sources have cores that are more prominent at high frequency. 


\section{(b) Active galactic nuclei}

Quasars are really just the brightest members (with powers in excess of $c a .10^{37} \mathrm{~W}$ ) of a larger class of 'active galactic nuclei' (AGN) (circumventing some ambiguities). In fact, it appears that the majority of 'normal' galaxies exhibit some form of nuclear activity. One particularly important type of AGN is the Seyfert Galaxy, first identified as a class in the 1940s. Seyfert galaxies come in two basic types. Type 1 Seyferts (e.g. NGC 4151) exhibit both broad and narrow optical emission lines and powerful soft X-ray emission, whereas type 2 Seyferts (e.g. NGC 1068) only show the narrow lines directly and are weak soft X-ray sources. Seyfert galaxies are often considered to be the low power extension of the quasar luminosity function, but there are several important differences: they are never powerful radio sources; they appear to be associated mostly with spiral galaxies while quasars may reside in giant ellipticals; Seyfert 1 galaxies are more powerful in X-rays relative to UV emission; and Seyferts never show very broad absorption lines.

\section{(c) Radio galaxies}

Powerful radio sources were first identified with giant elliptical galaxies in the 1950s. Like the quasars they can be extended (e.g. Cygnus A) or core-dominated (e.g. BL Lac, the eponymous 'blazar'). These radio sources are supplied with energy, momentum and magnetic field through a pair of jets that emerge from a source smaller than the compact radio components. The extended radio galaxies are further divided into the weaker 'Fanaroff-Riley' (FR) class 1 objects like Centaurus A and the more powerful FR2 objects like Cygnus A. It is found that the brightest radioemitting region is located at the extremities of the source in FR2 radio galaxies but near the centre of the source in FR1s.

\section{(d) Unification}

If all of this sounds a bit confusing, it should. In fact, the classification of AGN is much more complicated than I have described. (The subject is closer in spirit to clinical psychology than elementary particle physics!) However, there has been some progress in bringing order to the field through a process called 'Unification'.

There are at least four types of unification that have been examined. The best established is that compact radio sources are extended radio sources viewed along their relativistic jets. Essentially what we are seeing is the relativistically Dopplerboosted emission from the innermost parts of the jet outshining the unboosted emission from the surrounding extended radio source. (We know that relativistic motions are present in the compact cores, because radio astronomers can image features moving across the sky with apparent 'superluminal' speed.) This explains why, when we observe compact radio sources at low radio frequency, we see faint low surface brightness halos surrounding the compact source.

Almost as well established is the notion that Seyfert 2 galaxies are similar to Seyfert 1 galaxies except that they are observed through a warped equatorial disc, or torus, that prevents direct view of the broad emission lines and UV-soft-X-ray spectrum. Here the confirmation is provided by detection of broad emission lines from Seyfert 2 galaxies in polarized radiation. This has, presumably, been scattered in our direction so as to avoid the disc. 
Thirdly, it appears to be the case that many of the powerful FR2 radio galaxies are actually radio-loud quasars that would be classified as such if we were not viewing them through an obscuring dusty gas.

Finally, there is fairly good evidence that most radio-quiet quasars produce radiatively driven equatorial outflows and we only classify them as BALQ when our line of sight intersects these flows.

\section{Observations of black holes}

\section{(a) Black holes as prime movers of nuclear activity}

Ever since quasars were first discovered in 1963, black holes have provided one of the most popular explanations for their activity (see, for example, Lynden-Bell 1969). They naturally produced high radiative efficiency, rapid variation, long-term source axes and relativistic outflow speeds as the observations required. However, it is only in recent years that the positive observational evidence for the presence of black holes in the nuclei of the majority of regular galaxies has become overwhelming. As with stellar-sized black holes, the only sure approach is dynamical. Both stars and gas have had their speeds measured and the combination of speed and size suffices to estimate the central mass. It has been possible to measure about 25 hole masses. These range all the way from $c a .10^{6} M_{\odot}$ to $c a .3 \times 10^{9} M_{\odot}$ and have been localized in volumes that, in several cases, are too small to allow a long-lived star cluster. (The most celebrated is now NGC 4258 (Moran, these issue), which has a mass of $3.9 \times 10^{7} M_{\odot}$.) Beyond all reasonable doubt, these are black holes. In other words, this part of the story is on much firmer footing than the rest of what I shall say.

\section{(b) The galactic centre}

A good example is our galactic centre. Recently, Ghez et al. (1998) and Genzel et al. (1997) have been able to measure the motion of stars close to the hole's location, as determined by radio astronomy. As they are also able to measure the speed of the stars along the line of sight, it will soon be possible to reconstruct their individual orbits and verify directly that the central mass is point-like. The existing data are consistent with a black hole of mass $2.6 \times 10^{6} M_{\odot}$ and essentially nothing else. One important feature of the galactic-centre black hole is that it is surprisingly underluminous relative to the gas supply. Specifically, it seems that $c a \cdot 10^{19} \mathrm{~kg} \mathrm{~s}^{-1}$ of gas is supplied to the black hole. However, the bolometric luminosity appears to be not much more than $c a .10^{29} \mathrm{~W}$ and the quotient gives us $c a .10^{10} \mathrm{~J} \mathrm{~kg}^{-1} \sim 10^{-7} c^{2}$ : hardly a good advertisement for gravity power!

\section{(c) $M 87$}

Another good example is M 87, a galaxy in the Virgo cluster. Here, the speed of the gas orbiting the black hole is measured and implies a large black-hole mass, ca. $3 \times 10^{9} M_{\odot}$ (see Richstone et al. (1998) for a general review). Again, both the power and radiative efficiency are found to be low. M 87 is also a FR1 radio source with a single jet inclined at an angle $c a .30^{\circ}$ to the line of sight. (There is, presumably, a counter-jet that is rendered invisible by Doppler beaming.) Despite its low power, features have been reported to be moving with apparent speeds of $c a .6 c$, perhaps 
associated with some relativistic gas stream deflected slightly closer to the line of sight. In a triumph of precision astronomy, Junor \& Biretta (1995) have traced this jet to $c a .100 \mathrm{~m} \sim 5 \times 10^{11} \mathrm{~km}$ from the hole. This is the best direct evidence that we have that relativistic jets are formed close to black holes.

\section{(d) Spin}

The mass $m$ of a black hole, expressed in geometrical units, just determines a scale of length and time. Of more physical interest is a second parameter which measures the shape of the surrounding space-time. It is convenient to choose this to be the spin angular velocity of the hole, $\Omega$. This has a maximum value $1 / 2 m$, corresponding to an extreme Kerr hole. The most convincing case presented to date for having measured this quantity has been given for MCG 6-30-15 (Tanaka et al. 1995), where the shape of the measured ca. $7 \mathrm{keV}$ Fe line profile is similar to that expected to be produced by an accretion disc extending down to its limiting least-stable circular orbit from a hole that is spinning nearly maximally. Unfortunately, this is not the only interpretation of this profile (Reynolds \& Begelman 1997).

\section{(e) X-ray binaries}

Although these are not quasars, there are at least nine X-ray binaries where the mass of the compact object exceeds the maximum mass of a neutron star or white dwarf. Particularly prominent among these objects are the microquasars (Mirabel, this issue). Several of these sources exhibit 'quasi-periodic oscillations' (QPOs), presumably originating from short-lived disc modes. The frequencies of these modes must measure the hole mass and spin, though it has not yet been possible to explain how in a convincing manner.

\section{Observations of jets}

\section{(a) The gamma-ray revolution}

The general existence of jets, similar to those previously observed in $\mathrm{M} 87$ and 3C 273, was inferred in extragalactic double radio sources from the demonstrated need for a continuous supply of energy and linear momentum (Rees 1971). Bipolar outflows are now known to be a common feature of accreting objects; specifically, they have also been found in association with microquasars, young stellar objects and neutron star binaries. Until about eight years ago, this subject was the almost exclusive province of the radio astronomer. However, with the success of the EGRET instrument on Compton Gamma Ray Observatory (Hartmann et al. 1999), it has become apparent that the radio emission is often, and probably always, a bolometrically insignificant part of the jet luminosity. (The great strength of radio observations is that they enable us to image jets directly in fine detail.)

Extragalactic jets (see, for example, Hughes 1991; Ostrowski et al. 1997) present the cleanest challenge to astrophysicists. Let us draw together the evidence from several well-studied black-holes sources and gamble that they are fundamentally similar structures. We can then formulate a general model of jet formation and collimation. From the M 87 observation, it appears that jets are formed as collimated ultrarelativistic outflows on scales smaller than $c a .100 \mathrm{~m}$. Their initial composition 
is not known, but they quickly become prodigious emitters of GeV gamma-rays and are variable on time-scales as short as ca.30m (Wehrle et al. 1998). If we correct for relativistic kinematics, then the $\mathrm{GeV}$ gamma-ray emission region is probably located at radii less than $c a \cdot 10^{3} \mathrm{~m}-10^{4} \mathrm{~m}$.

An important energy-dependent radius is that of the ' $\gamma$-sphere', where the optical depth for a gamma-ray to create pairs by combining with X-rays is unity (Blandford \& Levinson 1995). A second important radius is the 'annihilation radius', within which electrons and positrons can cool to subrelativistic energies and annihilate in one expansion time. If efficient particle acceleration occurs between the annihilation radius and the $c a .0 .5 \mathrm{MeV} \gamma$-sphere, then the jet is likely to comprise electronpositron pairs at larger radii. (Whether or not there is evidence for this is an interesting controversy at the present time (Wardle et al. 1998).) However, the inner jet cannot comprise only pairs. There must be a second agency to carry the momentum and to overcome radiative drag so that the jet can persist to larger radii, as observed. The two candidates are the electromagnetic field and protons, with the former being preferred because any scheme to create a directed proton beam would probably require invoking an even larger electromagnetic energy density.

The gamma-ray spectrum extends up to greater than ca. $1 \mathrm{TeV}$ (Quinn et al. 1996) and is produced by inverse Compton scattering of soft photons that are probably created within the jet by the synchrotron process, although they may also be part of the ambient radiation field. ( $\mathrm{TeV}$ sources are rapidly variable but can only be observed out to $z \lesssim 0.1$, because of absorption on the cosmic infrared background.) The inner jet must therefore be capable of accelerating electrons to energies greater than $c a .1 \mathrm{TeV}$. It is hard to estimate accurately the jet beaming factor and efficiency and the amount of obscuration, but it appears that a typical ultrarelativistic jet carries a time-averaged power that is a significant fraction, perhaps a few per cent, of the total emitted power of the underlying quasar or AGN. In a few cases (e.g. Cygnus A), this fraction may be more than a half.

\section{(b) Radio observations}

At larger jet radii, $c a \cdot 10^{5} \mathrm{~m}-10^{6} \mathrm{~m}$, the outflow is essentially adiabatic. Initially, the radio synchrotron emission is self-absorbed and unresolved - the radio core. However, at larger radii, the emission is optically thin and radio astronomers are able to track the motion of relativistic shocks, the superluminally expanding features, travelling along the jet, accelerating high-energy electrons as they go. Optical and $\mathrm{X}$-ray synchrotron emission are observed out to quite large radii $\left(c a .10^{7} \mathrm{~m}\right)$ in a few sources (e.g. M 87), implying that these shocks are capable of accelerating ca. $100 \mathrm{TeV}$ electrons.

We know enough about the physical conditions in the radio-emitting regions to place a lower bound on the internal pressure and to compare this with the maximum external gas pressure at the same radius, deduced on the basis of X-ray observations. In the most powerful sources, the jet appears to be overpressured by factors of ca. 10-100. This disparity provides one of the strongest reasons for invoking magnetic collimation and confinement of jets.

A major uncertainty in our understanding of jets is the bulk Lorentz factor of the outflow, $\Gamma$. This is important because the observed flux density from a coherent feature moving towards us increases proportionally to $\Gamma^{3}$ at the transformed 
frequency, and so a relatively insignificant part of a poorly collimated outflow can outshine all of the rest of the jet for selected observers. (Note that $\Gamma$ refers to the motion of the emitting material, not the motion of the peak of the emission. In a shock, these are distinct.) Furthermore, although it has long been thought that the observation of superluminal expansion speeds less than $c a$. $10 c$ suggested that $\Gamma \lesssim 10$, typically we are now beginning to suspect that values an order of magnitude higher may be present. This is because of the discovery of intraday variability of the centimetre emission in several sources. If this is intrinsic to a synchrotron source, it implies that $\Gamma \gtrsim 1000$ in some cases and requires unreasonably large jet powers (Kedziera-Chudzczer et al. 1997). A more reasonable explanation of these variations is refractive scintillation in our interstellar medium. However, there may still be a problem because scintillation cannot change the source brightness temperature, which has been measured to be as large as $c a .10^{14} \mathrm{~K}$, two orders of magnitude in excess of the value needed to match the inverse Compton emission to the synchrotron emission. As brightness temperatures are boosted by one power of the Doppler factor in relativistic expansion, this suggests that $\Gamma \gtrsim 100$. A second reason for considering larger Lorentz factors is that the precedent has already been set by models of the most energetic gamma-ray bursts which suggest that they are beamed towards us with $\Gamma \sim 300$. All of this discussion has prompted a re-examination of coherent emission mechanisms which are not subject to these constraints, though are subject to other limitations.

\section{(c) What do we know about jet magnetic fields?}

Radio polarization observations indicate that the magnetic field in a jet is relatively ordered. On arc second scales, a characteristic pattern is observed with the more powerful FR2 radio galaxies exhibiting a parallel magnetic field while the less powerful FR1 sources show perpendicular magnetic fields (although parallel fields are sometimes seen at small radii and at the outer edges of resolved jets). The interpretation is straightforward. The magnetic-field direction reflects the rate of shear of the velocity field, with the parallel case arising when there is a significant velocity gradient across the jet or in a boundary layer and the perpendicular field predominating when the transverse expansion is most important, that is to say after internal velocity gradients have been erased. Note that we are supposing that the mean field component along the jet is very small. This is reasonable on general grounds because the associated magnetic flux would otherwise be very large and, as it is only likely to decrease along the jet, it would be associated with an unreasonable magnetic pressure close to the black hole.

There are also patterns on the milliarc second scales probed by VLBI observations (see, for example, Gabuzda et al. 1999). These show similar patterns to observations on larger angular scales and are also consistent with emission from travelling internal shocks.

\section{(d) Do discs launch hydromagnetic jets and, if so, how?}

Having described the magnetic field that we observe directly, what about the field that we cannot see? There are three distinct processes to which it may be contributing: launching of a powerful outflow close to the hole, collimation of this outflow into the narrow jets observed at somewhat larger distance and confinement 
of these jets on all scales out to the extended radio components. We have already attributed confinement on VLBI scales to magnetic stress. Can invisible magneticfield lines, initially frozen into a highly conducting accretion disc close to the black hole, wrapped around the jet, and each other, by the differential rotation, do the rest of the job?

Several general collimation mechanisms have been described (see Pudritz, this issue; Mestel 1998; Königl \& Pudritz 1999, and references therein). In particular, if there is a strong ordered magnetic field, the tension associated with its azimuthal component creates a collimating and confining 'hoop' stress. The gradient in the magnetic pressure may help. Magnetic field attached to an accretion disc also provides a means of launching a jet because it will exert a torque on the disc and extract some of the binding energy released by the infalling gas. A hydromagnetic wind may also remove a significant fraction of the accreting mass because, if the field direction subtends an angle of more than $\pm 30^{\circ}$ to the rotation axis, then gas will be flung away from the disc by centrifugal force. The resulting collimated hydromagnetic outflow is likely to have an asymptotic speed a few times the escape velocity at the magnetic footpoint on the disc. This elementary mechanism is straightforward to describe and analyse using similarity methods (see, for example, Ostriker 1997).

Another type of ordered MHD outflow model has been developed by Shu et al. (1994), more specifically for application to young stellar objects (see Clarke \& Pringle, this issue). Here, it is proposed that essentially all of the magnetic flux in the magnetosphere emanates from the innermost radii of the disc. One concern with this model is that some of these field lines must lie on the surface of the accretion disc and be subject to rapid reconnection as they sweep by loops and prominences attached to the disc. If this happens, the flux will migrate radially outward very quickly.

An organized field need not be unipolar. Lynden-Bell (1996) has developed quasistatic force-free models in which a poloidal magnetic loop is twisted by a differentially rotating disc and rapidly expands outwards creating toroidal field of one sign. One possible problem with this mechanism is that it is assumed that the Alfvén speed $v_{\mathrm{A}}$ is infinite, whereas, in practice, matter is likely to be flung out as well so as to lower the Alfvén speed below the rotational speed close to the disc and the outflow velocity far from the disc. However, the footpoints of a given loop will be differentially rotating and although the field line attached to the inner footpoint trails, the field line at the outer footpoint leads. Now a leading field line looses causal contact with the disc above a height $c a . v_{\mathrm{A}} / \Omega$ where it must be dragged by the returning field from the inner footpoint. The whole loop must therefore be sub-Alfvénic. Loops that become super-Alfvénic are presumably unstable and will shock, reconnect and detach.

Magnetic field can also be responsible for powering jets in a less organized manner. As discussed by Brandenburg (this issue), the magnetorotational instability drives a non-helical dynamo (Balbus \& Hawley 1998) and ensures that accretion discs are able to regenerate radial and toroidal magnetic field on an orbital time-scale and build up an internal disc field that is supposed to be much stronger than any ordered vertical field that leaves the disc surface. Under these circumstances, loops of field of a size comparable with the disc thickness (or more relevantly, the pressure scale height) will be continuously released by buoyancy and reconnection from the disc surface into an active corona in much the same way as is envisaged to happen at the surfaces of stars and the galactic disc (Miller \& Stone 2000, and references therein). Not only does 
this process seem unavoidable, it also provides a suitable power source for the X-ray emission of Seyfert galaxies and other AGN. However, it does not automatically lead to a collimated outflow. Tout \& Pringle (1996) have suggested that these small loops can grow through an inverse cascade to a size approximating $r$ and that these larger loops can provide enough tension to effect collimation despite the reversals in the sign of the azimuthal field with cylindrical radius and the isolation from the underlying rotating disc. In an alternative description, Heinz \& Begelman (2000) have suggested that the field is disordered on small scales and that its dynamical effect may be approximated by a local mean stress tensor. In a hybrid model (Blandford \& Payne 1982; Emmering et al. 1992), the RMS coronal field is supposed to be larger than the mean vertical field and constantly changing on time-scales shorter than an orbital period through reconnection. This allows matter to be injected, intermittently, onto open field lines and flung out into a wind where the field becomes relatively organized and smoothly varying.

A key difference in the underlying approach of these models is whether the mean field at high altitude is unipolar and established through a balance between advection by the inflowing disc from large radius (where most of the flux resides) and escape through reconnection, or whether it is created locally by dynamo action so that the horizontal correlation length is approximately less than $r$. What happens is unclear. On the one hand, van Ballegooijen (1989) argued that the advection rate is slower than the escape rate by a factor $O\left(r / H P r_{\mathrm{m}}\right)$, where $H$ is the disc thickness and $\operatorname{Pr}_{\mathrm{m}}$ is the effective magnetic Prandtl number. Therefore if $\operatorname{Pr}_{\mathrm{m}}<r / H$, and it is traditionally set to unity in a turbulent medium, then any large-scale field must escape. Alternatively, we can express the mean inflow rate in the disc as $t_{\text {in }}^{-1} \sim$ $\alpha \Omega(H / r)^{2}$, where $\alpha \sim 0.1$ is the assumed coefficient of proportionality between the shear stress and the pressure, and observe that, to within a numerical factor of order unity, this is the rate at which the magnetic field will random walk out of the disc by reconnecting on flux loops of approximate size $H$ every $\Omega^{-1}$.

Of course, the polarity of the field associated with the accreting gas is likely to change. This does not preclude confinement by the hoop stress associated with the toroidal field, for which the polarity must also change. To see this, consider an elementary model in which an axial current $I$ flows along a jet. There is an axisymmetric toroidal field of strength $\mu_{0} I / 2 \pi r$. Now let there be axisymmetric axial currents of strength $2 I$ and alternating sign flowing within thin cylindrical sheaths of radius $r_{1}, r_{2}, \ldots$. The toroidal field magnitude will be unchanged but the sign will reverse. The stress acting on the current sheets $\mu_{0} I^{2} / 8 \pi^{2} r_{i}^{2}$, will be balanced across them and will steadily decrease until it can be matched onto an ambient gas pressure. Stress balance within the current sheets must be achieved either with gas pressure or a rotating magnetic field. This configuration is presumably tearing mode unstable and magnetic energy will be steadily dissipated through reconnection. However, it should persist for long enough in a super-Alfvénic outflow to allow jet collimation.

As must be clear by now, this is a contentious subject. As has also been true of cosmology, there are several different elementary models that are amenable to applied mathematical analysis without any guarantee that their underlying assumptions are relevant to the application. We simply do not understand MHD well enough yet to know what are the correct assumptions to use and this is a prerequisite to answering the big astrophysical questions. We need to know the ratio of the internal torque transporting angular momentum radially outward through the disc to the external

Phil. Trans. R. Soc. Lond. A (2000) 
torque applied to the disc surface and responsible for carrying off angular momentum in the wind. (Note that if the mean vertical field threading the disc is large, then the magnetorotational instability is likely to be suppressed.) Furthermore, we want to understand the magnetic structure and energy balance of the disc corona. Presumably it is a low $\beta$ plasma which can be approximated as force free, in contrast to the disc field. Stability is another issue. For example, simple prescriptions for specifying the rate at which mass is loaded onto open field lines lead to the conclusion that a centrifugally driven wind is unstable (Lubow et al. 1994); alternative prescriptions lead to stationary self-adjusting flows (Königl \& Wardle 1996; Krasnopolsky 2000, unpublished work). Another difficult issue is the nature of the boundary conditions to apply at large and small cylindrical radius. In a similarity solution the difficulty is finessed. However, in a finite disc the ultimate collimation can be strongly influenced by what is assumed (Okamoto 2000, and references therein).

However, the situation is not hopeless. There are at least three lines of inquiry that are helping a lot. Before I explain why, though, I would like to discuss two further relevant questions.

\section{Is adiabatic accretion conservative?}

In recent years, there has been renewed interest in what happens when gas accretes at a slow rate or, more specifically, at low density (relative to Eddington accretion). Under these circumstances there is the possibility that the flow is adiabatic (in the sense that it does not cool on a dynamical time-scale). This can surely happen if the only coupling between the ions and the electrons is through Coulomb scattering. When the mass accretion rate in units of the Eddington rate $\left(4 \pi G M / c \kappa_{T}\right)$, denoted $\dot{m}$ is greater than $c a .0 .3 \alpha^{2}$, then cooling will be ineffective and the disc is likely to inflate as a consequence of its large internal energy. There has been a lot of work in recent years describing conservative flows (called ADAFs (see, for example, Narayan et al. 1998; Kato et al. 1998, and references therein)) that carry all the supplied mass down the black hole with negligible radiative loss. Fitting the emissivity computed from these flows to diverse observed sources has been a relatively successful enterprise.

However, there are some fundamental dynamical problems with ADAF solutions. The gas is formally unbound, mainly because the viscous torque that allows it to proceed transports energy as well as angular momentum and this must be dissipated in a differentially rotating disc. To be specific, if there is an extensive adiabatic conservative viscous disc flow, then it can be shown that the specific energy of the gas is twice its orbital kinetic energy. The model, as it stands, does not appear to be self-consistent without becoming quasi-spherical and then the gas close to the rotation axis is unsupported.

For these and other reasons, Blandford \& Begelman (1999) have proposed that adiabatic accretion should always be accompanied by outflows which carry off energy, angular momentum, mass, etc., in unspecified amounts that are sufficient to allow the gas to accrete on bound orbits. The outflows may be gas dynamical or hydromagnetic. In these 'ADIOS' solutions, the rate at which gas actually accretes onto the black hole can be orders of magnitude less than the rate at which it is supplied. If this view of adiabatic accretion ultimately prevails, and there are some ways by which

Phil. Trans. R. Soc. Lond. A (2000) 
it can be distinguished observationally from ADAF flows, then there will be a good dynamical reason why accretion is often accompanied by outflow.

A second mode of adiabatic accretion can occur when the gas is supplied at a rate far in excess of the Eddington rate $(\dot{m} \gtrsim 10)$. Under these circumstances, there is no difficulty in emitting radiation. The problem arises when the photons try to escape (Begelman \& Meier 1982). It turns out that they will be trapped in the accreting gas and advected in toward the hole faster than they can diffuse away. Again the flow is likely to be effectively adiabatic and is likely to drive an outflow for the same reason as a subcritical inflow. If this view turns out to be correct, it will be hard for black holes to accrete mass at a rate that is much larger than roughly ten times the Eddington rate. These outflows, launched initially by Thomson scattering and then further accelerated by resonance line scattering may be associated with the absorbing gas in BALQs.

\section{Are quasar jets powered by black-hole spin energy?}

When a black hole spins and its space-time is described by the Kerr metric, a fraction less than or equal to 0.29 of its mass energy can be associated with its spin and is extractable. A Gedankenexperiment to do just this was performed by Penrose (1969). For the $c a .3 \times 10^{9} M_{\odot} \equiv 5 \times 10^{56} \mathrm{~J}$ black hole in M 87, perhaps $c a .10^{56} \mathrm{~J}$ of energy can realistically have been tapped over its life, which is ample to account for an extremely profligate youth.

\section{(a) How to get blood out of a stone}

The most natural way to tap this energy is by using a large-scale magnetic field (Blandford \& Znajek 1977; Thorne et al. 1986; Lee et al. 2000). Currents flowing in the inner accretion disc can support a significant amount of magnetic flux (typically $\Phi \sim 10^{25} \mathrm{~Wb}$ threading the hole. Now the hole can be considered to be a good, though not perfect, electrical conductor, with a surface conductance of $(E / H)_{\text {horizon }}=Z_{0}=377 \Omega$. Therefore, when the hole spins, it can act as a unipolar inductor and create an EMF $\mathcal{E} \sim \Omega \Phi \sim 10^{20} \mathrm{~V}$ (just about sufficient to accelerate the UHE cosmic rays). This EMF can drive a closed field-aligned current circuit that dissipates both within the horizon of the hole (the internal resistance of the circuit) and in the particle acceleration region at the base of the jet (the load). As the total resistance of the circuit is $c a .100 \Omega$, the current, in this example, is $c a .10^{18} \mathrm{~A}$ and the power is $c a .10^{38} \mathrm{~W}$. The power can be thought of as being transported away from the horizon in the form of a Poynting flux. (The 'no-hair' theorem is not violated because the flux of energy is only conserved in the frame that is not rotating with respect to infinity. Observers that hover just above the horizon must rotate with respect to this frame and they would observe an inwardly directed energy flux.) The electromagnetic power scales according to the memorable relation $L \propto a^{2} B^{2} c$, where $B$ is the field that threads the hole. (This stipulation is important because magnetic flux is unable to penetrate the horizon when the rotation rate is nearly maximal and so the electromagnetic power is reduced.)

This mechanism has recently attracted attention because of its possible role in powering gamma-ray bursts. The magnetic field is quite likely to be separated from the accreting gas so that the resulting outflow can move ultrarelativistically. Similar,

Phil. Trans. R. Soc. Lond. A (2000) 
though less extreme, conditions are required in quasar jets. However, it has also been argued that the power extracted from the hole is likely to be much less than that extracted hydromagnetically from the inner disc, mainly because the area of the latter is larger (Livio et al. 2000). This is probably true, at least for a thin disc (see Blandford \& Znajek 1977). However, quasar jet powers are only a fraction of the bolometric power, as a comparison of the gamma-ray background with the quasar light background affirms and they can still have a black-hole origin. Furthermore, in a thick disc, perhaps associated with an adiabatic inflow, a funnel can form and the jet-power fraction can plausibly become quite large (see, for example, Rees et al. 1982).

Another objection has been put forward by Natarajan \& Pringle (1998) who have presented a new analysis of the Bardeen-Petterson mechanism whereby a spinning hole will interact dynamically with a misaligned outer disc. They conclude that black holes will align more rapidly than previously estimated and, if the plane of the gas supply keeps changing, the hole will spin down faster than accretion will cause it to spin up. (Note that this alignment is coupled with a quite large release of energy in the outer disc $O\left(m^{3} \Omega \omega_{\mathrm{BP}} \theta^{2}\right)$, where $\omega_{\mathrm{BP}}$ is the Keplerian angular velocity at the warp radius and $\theta$ is the misalignment between the hole spin and the outerdisc angular momentum.) The estimated alignment time-scales typically fall between the jet transit times to the outer lobes of radio sources and the overall radio source lifetimes consistent with the 'dentist's drill' model of lobe advance. VLBI observations are already resolving scales at approximately $r_{\mathrm{BP}}$ and may soon determine if the jet axis is determined by the hole or the disc.

Despite all these concerns, there is still a particularly good reason for invoking the extraction of electromagnetic energy from a spinning hole to power quasar and similar jets. This is because, as I have emphasized, they are ultrarelativistic and, initially, probably magnetically dominated. The hydromagnetic winds from the surface of a disc are unlikely to avoid being loaded with plasma and, consequently, will be unlikely to achieve high terminal Lorentz factors. No such drawback attends the field lines that thread the surface of a black hole!

\section{(b) Flogging a dead horse}

In a variant of this mechanism, that has also just been resurrected, it may be possible to transfer angular momentum from the hole directly to the disc. Krolik (2000) and Gammie (2000) have considered the magnetic field in the plane of the disc within the innermost stable circular orbit and shown that it is possible for magnetic torque to carry an energy flux outward along a magnetic-flux tube, at least outside the ingoing Alfvén critical point and, in this manner, increase the specific energy release from the disc to a value above that associated with the binding energy at the innermost stable circular orbit. The extra power is, again, derived from the spin of the hole. One particular concern about this mechanism is that the infalling gas remains magnetically attached to the disc. It seems quite likely that both senses of radial field will be present within the transition region between the disc and the hole and that magnetic reconnection will happen quite freely. An alternative way to model this interaction (Blandford \& Spruit 2000), is to suppose that magneticflux tubes connect the disc surface to the event horizon at intermediate latitude, extracting energy and angular momentum from the hole. Note that, in this case, the

Phil. Trans. R. Soc. Lond. A (2000) 
magnetic field is tied to the orbiting gas not the central object - the opposite of what happens with accreting neutron stars.

\section{Laboratories for studying MHD}

As I have already emphasized, we are seriously handicapped by our ignorance of how the magnetic field actually behaves in cosmic environments. Fortunately, there are three promising approaches to improving our understanding.

\section{(a) Cosmic laboratories}

The first of these is direct observation of dynamical magnetic fields.

\section{(i) Solar wind}

Observations of the solar photosphere and corona by Yohkoh, SOHO and TRACE, as reported by Title (this issue), have led to a revolution in our general perspective on MHD. No longer is the Sun observed as having a bland surface occasionally ruptured by coronal arches. Instead, it is a tightly interwoven largely invisible tapestry of moving magnetic field, energized by the underlying convection and held together for as long (typically 1-2 d) as reconnection and intercommutation of magnetic-field lines can be staved off. The observed corona is maintained at a temperature of ca. 1$2 \times 10^{6} \mathrm{~K}$, probably by reconnecting nanoflares, though the detailed calorimetry is still a matter of controversy. The magnetic field dominates the energy density. By contrast, $\beta>1$ below the photosphere, where it has just been found that a dynamo may be operating, in addition to the dynamo located at the base of the convection zone that produces the field of sunspots.

Observations during solar minimum of the high-latitude wind at ca.1-2 AU by Ulysses have been no less instructive (see, for example, Fisk 1998). The wind and its associated flux emanate from giant coronal holes located near the poles. The radial magnetic field at $1 \mathrm{AU}$ has a steady value of $c a .4 \mathrm{nT}$ and satisfies an inverse-square variation with radius indicating that it more or less fills most of a hemisphere in a uniform manner. The total field pattern executes a Parker spiral, although it is slightly overwound at the poles. The density is $c a .3 \times 10^{-21} \mathrm{~kg} \mathrm{~m}^{-3}$ and again is pretty constant with time and latitude, satisfying an inverse-square variation with radius suggesting that the surprisingly large and uniform outflow speed of $c a .800 \mathrm{~km} \mathrm{~s}^{-1}$ is also achieved well before ca. 1 AU.

The wind cannot be thermally driven; instead it is supposedly energized by Alfvén waves close to the corona. We can actually use this information to estimate the torque that the solar wind exerts upon the Sun at this time. If we approximate the wind as spherical and the radial velocity as pretty constant, then the torque is given by

$$
G \sim 2 \pi r^{4} v_{\mathrm{r}} \rho \Omega \sim \frac{2 \pi B_{\mathrm{r}}^{2} r^{4} \Omega}{\mu_{0} v_{\mathrm{r}}}
$$

evaluated at the Alfvén radius, where $v_{\mathrm{r}}=B_{\mathrm{r}}\left(\mu_{0} \rho\right)^{-1 / 2}$. This is given by $G \sim$ $10^{23} \mathrm{~N} \mathrm{~m}$ and the Alfvén radius evaluates to $c a .12 R_{\odot}$. The solar angular momentum is, for comparison, ca.1.6 $\times 10^{41} \mathrm{~kg} \mathrm{~m}^{2} \mathrm{~s}^{-1}$ and so the characteristic slowing down time is now ca. $30 \mathrm{Gyr}$, consistent with expectation. 
A more sophisticated comparison is certainly possible and would have to take into account that the coronal hole axis is inclined with respect to the spin axis, that there is a separate slow wind at low latitude, that there is a significant amount of Alfvén turbulence, that the Sun differentially rotates, that there is a solar cycle and so on (see Fisk 1996). However, even though the Sun is a slow rotator relative to an accretion disc, it clearly has much to teach us as we try to model hydromagnetic winds from accretion discs. In particular it may already have told us that simple stationary axisymmetric models of disc winds are a good starting point.

\section{(ii) Crab Nebula}

Recent HST observations of the Crab Nebula also have some lessons for us. The main reason is that a similar path is being followed by the energy:

ordered rotational energy $\rightarrow$ electromagnetic energy

$\rightarrow$ relativistic electron energy $\rightarrow$ non-thermal radiation.

Moving features have been observed associated with the famous wisps, which may coincide with the strong relativistic shock formed where the momentum flux in the outflow matches the ambient nebula pressure (Gallant \& Arons 1994). Our understanding remains somewhat sketchy, but this is the closest we are ever likely to be to particle acceleration in an ultrarelativistic plasma, so it is worth persisting. It ought to be possible to understand the speed and composition of the outflow and whether or not a strong collisionless shock is really formed. The absence of a narrow jet may well point to the importance of having an extended disc for forming jets.

\section{(iii) X-ray astronomy}

The next eight months will see the launch of three complementary X-ray telescopes: Chandra, XMM and Astro-E. They should improve our understanding of the structure of jets, discs and the cosmological distribution of quasars in much the same way that Yohkoh, SOHO and TRACE have so enriched our view of the Sun.

\section{(b) Numerical MHD laboratories}

The second laboratory is computational. As we have seen from Brandenburg (this issue, and references therein), the capability to perform relatively high dynamic-range three-dimensional numerical MHD is already here and there have already been serious attempts to expand this capability into the realm of special and general relativity. This facilitates a variety of numerical experiments. For example, Stone et al. (2000) have recently carried out a series of two-dimensional simulations of adiabatic accretion in which they consider purely hydrodynamical flows and introduce a variety of ad hoc prescriptions for the viscosity. They find that the gas becomes strongly convective with the mean specific angular momentum being constant on isentropes and the mean mass flow through radius $r$ settling down to a non-conservative variation, $\dot{M} \propto r$, consistent with the predictions of a limiting ADIOS solution. However, instead of creating a supersonic wind, the outflow is subsonic and is mostly confined to the surface of the disc. This highlights the fact that an extra source of entropy must be present at the disc surface for a disc to create a supersonic outflow purely 
hydrodynamically. Furthermore, by contrasting these simulations with their hydromagnetic counterparts (J. F. Hawley 1999, personal communication), it has become clear that the dissipation associated with the magnetic torque must be handled very carefully numerically and that the character of the flow may depend upon what is assumed. For example, the magnetic field may create a turbulence spectrum that is absorbed roughly volumetrically at some inner scale through transit time damping (Gruzinov 1998; Quataert 1998), or the entropy may be produced in a small fraction of the total volume if reconnection at current sheets dominates (Bisnovatyi-Kogan \& Lovelace 1997). Although in both cases the dissipation is local, the latter assumption is likely to lead to higher temperatures and a different emissivity than the former. Alternatively, most of the energy may be transported hydromagnetically from the disc to the corona so that there is little local dissipation. These assumptions may lead to quite different flows.

\section{(c) Plasma physics laboratories}

We have already mentioned several instances where magnetic reconnection can have a major role in determining the energy release and the details of the flow. As described by Parnell (this issue), we still do not have a confident understanding of this important process and some novel reconnection modes are currently under serious consideration. The way reconnection works in two dimensions is now well understood and the emphasis has shifted towards studying ways in which it may operate in three dimensions (see, for example, Priest \& Titov 1996; Galsgaard \& Nordlund 1997). Another way to approach this problem is through laboratory experimentation. Although the magnetic Reynolds numbers are never as high in the laboratory as one would like, it is still possible to perform instructive experiments and then to scale with the relevant dimensionless numbers (see, for example, Brown 1998) so as to learn how astrophysical reconnection occurs under differing conditions.

\section{What next?}

In this paper, I have tried to consider the problem of understanding powerful relativistic quasar jets in a general context. An outline of one solution, in which an electromagnetic core is collimated by a non-relativistic hydromagnetic wind, has existed for twenty years. However, it has not been verified satisfactorily observationally and there are several genuine physical difficulties that are not understood. There is still a chance that a quite different and essentially non-magnetic mechanism might be at work. However, as I have emphasized, the powerful combination of numerical simulation and direct observation of 'real' plasma is forcing us to become much more sophisticated and further important insights are likely over the next few years.

I would like to conclude by mentioning some speculative extensions of this model to a 'grand unified theory' of AGN that attempts to give a comprehensive interpretation of all of the principal modes of nuclear activity that are observed. This is stimulated by two recent observational claims. Firstly, Magorrian et al. (1998) have argued that the black-hole mass in local dormant galaxies is proportional to the mass of the 'bulge'. (Ellipticals are all bulge; spirals have progressively smaller bulges as the type changes from Sa to Sd.) Secondly, McClure et al. (2000) have presented evidence that quasars are surrounded by elliptical galaxies, not spirals as once thought.

Phil. Trans. R. Soc. Lond. A (2000) 
Suppose that black holes grow, as argued above, at an Eddington-limited rate early in the life of a galaxy. The rate of mass supply should decline with time, whereas the Eddington limit grows with mass. It is possible that the hole will grow with an e-folding time-scale $c a .30 \mathrm{Myr}$ until it reaches a mass somewhat below its present value. When the mass supply is super-Eddington, and the hole mass exceeds ca. $10^{8} M_{\odot}$, the object will form a radio-quiet quasar and produce a high-speed radiatively driven wind. During the final e-folding of hole mass, this wind will deposit $c a .10^{53}\left(\mathrm{~m} / 10^{8} M_{\odot}\right) \mathrm{J}$ of energy in the outer parts of the Galaxy and, presumably, will drive a blast wave into the infalling gas. If we assume that the escape velocity is $c a .300 \mathrm{~km} \mathrm{~s}^{-1}$, up to $10^{12}\left(\mathrm{~m} / 10^{8} M_{\odot}\right) M_{\odot}$ of gas can be driven away. Allowing for inefficiency and radiative loss, there is enough energy to expel the gas and forestall the formation of a disc if $m \gtrsim 10^{8} M_{\odot}$. In other words, relatively big black holes lead to elliptical galaxies. We know that BAL outflows are not seen when the luminosity is less than that associated with a quasar. (The explanation may be a subtle effect associated with opacity.) It is then possible that smaller holes cannot expel the infalling gas and that a disc will form. In other words galaxies form around black holes, not vice versa.

One key observation that must still be explained is that low-mass holes/spirals/ Seyfert galaxies do not create powerful ultrarelativistic jets. Perhaps accretion continues for much longer at an intermediate rate as the supply of gas is not shut down and this is sufficient to drive the spin of the hole to nearly its maximal value (as reported for MCG 6-30-15). This will prevent the hole from forming a powerful relativistic jet. Alternatively, the collimating hydromagnetic disc wind may just not be produced at an intermediate accretion rate. By contrast, with high-mass holes/ellipticals/quasars, the mass supply may quickly decline below Eddington and perhaps become adiabatic close to the hole. A radio-loud quasar or FR2 radio galaxy can then form. This will persist while the spin energy is depleted and the central jet becomes progressively less powerful. Eventually, the jet thrust becomes less than that associated with the disc wind and, although the observed jet may be formed with approximate speed $c$, it will soon be decelerated by interacting with the more slowly moving wind. This is an FR1 radio galaxy. If nothing else happens, the jet and nuclear activity will finally decline to dormancy. However, if two old galaxies and their black holes subsequently merge, a fairly rapidly spinning hole may ensue and a powerful radio source will be reborn (Wilson \& Colbert 1995).

These speculations have clear observable implications.

I thank The Royal Society for support and the other attendees for instruction and suggestions. I also thank participants in the Black Hole Astrophysics Program at the Institute for Theoretical Physics, Santa Barbara, notably, for many discussions of these and related topics. This research was also supported by the Beverly and Raymond Sackler Foundation, NSF grants AST 95-29170, 99-00866, PHY 94-07194 and NASA grant 5-2837.

\section{References}

Balbus, S. A. \& Hawley, J. F. 1998 Rev. Mod. Phys. 70, 1.

Begelman, M. C. \& Meier, D. L. 1982 Astrophys. J. 253, 873.

Bisnovatyi-Kogan, G. S. \& Lovelace, R. V. E. 1997 Astrophys. J. 486, L43.

Blandford, R. D. \& Begelman, M. C. 1999 Mon. Not. R. Astron. Soc. 303, L1.

Blandford, R. D. \& Levinson, A. 1995 Astrophys. J. 441, 79.

Phil. Trans. R. Soc. Lond. A (2000) 
Blandford, R. D. \& Payne, D. G. 1982 Mon. Not. R. Astron. Soc. 199, 883.

Blandford, R. D. \& Znajek, R. L. 1977 Mon. Not. R. Astron. Soc. 179, 433.

Brown, M. R. 1998 Bull. Astrophys. Soc. 43, 1661.

Emmering, R. T., Blandford, R. D. \& Shlosman, I. 1992 Astrophys. J. 385, 460.

Fisk, L. A. 1996 J. Geophys. Res. 101, 15547.

Fisk, L. A. 1998 Proc. 25th Int. Cosmic Ray Conf.

Gabuzda, D. C., Mioduszewski, A. J., Roberts, D. H. \& Wardle, J. F. C. 1999 Mon. Not. R. Astron. Soc. 303, 515.

Gallant, Y. \& Arons, J. 1994 Astrophys. J. 435, 230.

Galsgaard, K. \& Nordlund, A. 1997 J. Geophys. Res. 102, 231.

Gammie, C. F. 2000 Astrophys. J. (In the press.)

Genzel, R., Eckart, A., Ott, T. \& Eisenhauer, F. 1997 Mon. Not. R. Astron. Soc. 291, 219.

Ghez, A. M., Klein, B. L., Morris, M. \& Becklin, E. E. 1998 Astrophys. J. 509, 678.

Gruzinov, A. 1998 Astrophys. J. 501, 787.

Hartmann, R. C. (and 26 others) 1999 Astrophys. J. Suppl. 123, 79.

Heinz, J. \& Begelman, M. C. 2000 Astrophys. J. (Submitted.)

Hughes, P. A. 1991 Beams and jets in astrophysics. Cambridge University Press.

Junor, W. \& Biretta, J. A. 1995 Astronom. Jl 109, 500.

Kato, S., Fukue, J. \& Mineshige, S. 1998 Black hole accretion disks. Kyoto University Press.

Kedziora-Chudczer, L. et al. 1997 Astrophys. J. 490, 9.

Königl, A. \& Pudritz, R. E. 1999 In Protostars and planets IV (ed. V. Mannings, A. P. Boss \&

S. S. Russell). Tucson, AZ: University of Arizona Press.

Königl, A. \& Wardle, M. 1996 Mon. Not. R. Astron. Soc. 279, L61.

Krolik, J. H. 1998 Active galactic nuclei. Princeton University Press

Krolik, J. H. 2000 Astrophys. J. (In the press.)

Lee, H. K. Wijers, R. A. M. J. \& Brown, G. E. 2000 Phys. Rep. (In the press.)

Livio, M., Ogilvie, G. I. \& Pringle, J. E. 2000 Mon. Not. R. Astron. Soc. (In the press.)

Lubow, S. H., Papaloizou, J. C. B. \& Pringle, J. E. 1994 Mon. Not. R. Astron. Soc. 268, 1010.

Lynden-Bell, D. 1969 Nature 223, 690.

Lynden-Bell, D. 1996 Astrophys. J. 279, 389.

McClure, R. J. et al. 2000 Astrophys. J. (In the press.)

Magorrian, J. (and 11 others) 1998 Astronom. Jl 115, 2285.

Mestel, L. 1998 Cosmic magnetic fields. Cambridge University Press.

Miller, K. A. \& Stone, J. M. 2000 Astrophys. J. (In the press.)

Narayan, R., Mahadevan, R. \& Quataert, E. 1998 Theory of black hole accretion discs (ed. M. A.

Abramowicz, G. Björnsson \& J. E. Pringle), p. 148.

Natarajan, P. \& Pringle, J. E. 1998 Astrophys. J. 506, L97.

Okamoto, I. 2000 Mon. Not. R. Astron. Soc. (In the press.)

Ostriker, E. C. 1997 Astrophys. J. 486, 291.

Ostrowski, M. et al. 1997 Relativistic jets in AGNs. Kraków: Jagellonian University Press.

Penrose, R. 1969 Riv. Nuovo Cim. 1, 252.

Priest, E. R. \& Titov, V. S. 1996 Phil. Trans. R. Soc. Lond. A 354, 2951.

Quataert, E. 1998 Astrophys. J. 500, 978.

Quinn, J. (and 24 others) 1996 Astrophys. J. 456, L83.

Reynolds, C. \& Begelman, M. C. 1997 Astrophys. J. 488, 109.

Rees, M. J. 1971 Nature 229, 312.

Rees, M. J. et al. 1982 Nature 295, 17.

Richstone, D. O. et al. 1998 Nature 395, 14.

Robson, I. 1996 Active galactic nuclei. Chichester: Wiley. 
Shu, F. H. et al. 1994 Astrophys. J. 429, 781.

Silk, J. I. \& Rees, M. J. 1998 Astron. Astrophys. 331, L1.

Stone, J. M., Pringle, J. E. \& Begelman, M. C. 2000 Mon. Not. R. Astron. Soc. (In the press.) Tanaka, Y. et al. 1995 Nature 375, 659.

Thorne, K. S., Price, R. M. \& MacDonald, D. 1986 Black holes: the membrane paradigm. Yale University Press.

Tout, C. A. \& Pringle, J. E. 1996 Mon. Not. R. Astron. Soc. 281, 219.

van Ballegooijen, A. A. 1989 In Accretion disks and magnetic fields in astrophysics, p. 99. Dordrecht: Kluwer.

Wardle, J. F. C. et al. 1998 Nature 395, 457.

Wehrle, A. E. et al. 1998 Astrophys. J. 497, 178.

Wilson, A. \& Colbert, E. J. M. 1995 Astrophys. J. 438, 62.

\section{Discussion}

D. Lynden-Bell (Institute of Astronomy, Cambridge, UK). Thank you. A grand survey, but I was a bit disappointed that the greatest unification you talked about did not include the jets from young stellar objects and did not include the origin of the gamma-ray bursts. I regard these as part of one whole gamut-maybe you don't!

R. D. Blandford. It was on my first slide, but I think I omitted to emphasize that point. It is very much the modern way to look for the similarities between the dynamics of the Galactic superluminal sources you heard about earlier and the young stellar objects. We are learning lessons. Obviously, the young stellar objects are not relativistic, but nonetheless there is the dynamical equivalent of the central black hole there which may be responsible for creating the optical jets. So we do think about it that way.

S. FALle (University of Leeds, UK). The ADIOS solutions, as I understand it, rely on the accretion rate being dominated by the momentum loss from the wind. The viscous dissipation is not supposed to play any role in the disc. Is that right?

R. D. Blandford. No, not at all. If we're going to talk about these ADIOS solutions, the way that they actually work is that there is a fast convection along what's called the gyrentropes, which are surfaces of constant angular momentum. Then on a slightly slower time-scale, there's a magnetic transport of angular momentum outwards through the disc. The standard magnetic viscosity in an accretion disc is vital. Then on top of that we have a surface loss of mean angular momentum and energy in a proportion you can assign and that allows accretion to proceed on bound orbits.

S. FALLE. So the viscosity that is assigned to these numerical simulations was something based on what you guessed?

R. D. Blandford. In Jim Stone's case, he did numerical simulations and he had a Shakura-Sunyaev modified viscosity. He didn't allow for the vertical shear component.

I. F. Mirabel (CEA/SACLAY, France). There seems to be a universal correlation between the velocity in the last Keplerian orbit (escape velocity) around the central object and the velocity of fluid ejecta. This seems to hold from the Sun and young stellar objects (few hundreds of $\mathrm{km} \mathrm{s}^{-1}$ ), through white dwarfs in supersoft sources (few thousands of $\mathrm{km} \mathrm{s}^{-1}$ ), neutron stars $(0.2 c-0.3 c)$ and black holes $(>0.9 c)$. The 
Blandford-Znajek mechanism to power these jets would be exclusive of black holes, a sort of ad hoc hypothesis in the context of such a universal correlation. Could you comment on this?

R. D. Blandford. In the case of the compact radio sources one sees, by direct measurements, Lorentz factors of about 10, so that's not $90 \%$ of the speed of light, it's $99 \%$. The speed is even larger for the GRBs. For this reason one needs an ultrarelativistic source and there is none better than a black hole. It is much better than a corona at the inner edge of an accretion disc, where I think it would be very hard to produce such speeds. So a question I would put to you is why can't you find galactic superluminal sources moving at $0.99 c$ ?

I. F. Mirabel. Perhaps in our galaxy we have a gamma ray burst every 10 million years.

R. D. Blandford. I'm not talking about a gamma-ray burst, I'm talking about an accreting black hole as in $1915+105$. Why isn't $1915+105$ going at $0.99 c$, like its extra-galactic counterparts?

I. F. Mirabel. I think the major difference is that in the case of galactic black holes we observe recurring ejection events from instabilities that come ultimately from accretion instabilities in a disc, whereas, in the case of the gamma-ray burst with ultrarelativistic jets having Lorentz factors of 200, they are catastrophic events that do not repeat and are associated with the formation of a black hole. Perhaps we have to wait for 10 million years to have one such event in the Galaxy. That's my reading of the situation.

Y. UCHIDA (University of Tokyo, Japan). You mentioned magnetic coupling between the black hole and the disc so that angular momentum is given to the disc, but that doesn't help - it just quenches the gravitational energy release of the material falling into the back hole doesn't it?

R. D. Blandford. I would see it as a time-dependent phenomenon. The analogy is a roulette wheel: when the hole is spinning rapidly, the gas falls in its magnetic field which feels the black hole; that communicates angular momentum out to the disc at such a large rate that the gas then moves radially outwards through the disc; and later it falls in again. Obviously, each time it falls in, some of it accretes but the accretion may be time dependent.

A. Shukurov (University of Newcastle, UK). My question is about the magnetic fields in the jet. Given the opening angle, is the estimate of the field strength compatible with the assumption that the field is frozen into the flow? Is there any necessity to replenish the magnetic field within the jet?

R. D. Blandford. It depends where you think the field comes from. I have tried to present here a view that says the central object is separate from the collimating wind. Now at some level that's not right, there's a continuity between the two, but basically what we're doing is taking the equipartition fields or equivalently the powers in the jets and then inferring the magnetic fields on the accretion disc. We don't have an independent estimate of those fields. You heard a valiant attempt by Jim Moran at trying to measure the fields in the case of 4258 and all he found was an upper limit. Eventually, we may have a good estimate, but we don't have that as yet. So I can't answer your question at the moment. 
E. R. PRIEST (University of St Andrews, UK). You suggested four or five different MHD ways of collimating a jet. Do you believe they all work, or do you favour one in particular?

R. D. Blandford. Oh, no, I was just trying to give a little bit of a review. I didn't go through all of them. No, I believe the model in which most of the disc surface has closed field lines and a small fraction of the area has open field lines which eventually fill space is best. I personally think problems with collimating jets when you have fields that reverse (namely, avoiding reconnection and providing enough focusing) are so great that it's more reasonable to have one sign of flux at least for several decades of radius, just like in the solar wind. They can reverse after a while but I would say that for several decades of radius the field is ultimately derived from fields at larger radius and comes in rather than being produced by a local dynamo.

L. Mestel (The University of New Hampshire, USA). Is the collimation in your view due primarily to the toroidal field, or to the poloidal field, as argued, for example, by Spruit (Blandford \& Spruit 2000).

R. D. Blandford. Both play a role initially, although I tend to think the fields are ultimately toroidal, again as in the solar wind.

\section{Additional reference}

Blandford, R. D. \& Spruit, H. C. 2000 (In preparation.) 